\title{
Mild and efficient ring opening of monoterpene-fused $\beta$-lactam enantiomers. Synthesis of novel $\beta$-amino acid derivatives
}

\author{
Zsolt Szakonyi, and Ferenc Fülöp* \\ Institute of Pharmaceutical Chemistry, University of Szeged, H-6701 Szeged, POB 121, Hungary \\ E-mail:fulop@pharma.szote.u-szeged.hu
}

Dedicated to Professor Branko Stanovnik on his $65^{\text {th }}$ birthday

(received 17 June 03; accepted 20 Jan 04; published on the web 31 Jan 04)

\begin{abstract}
Both enantiomers of the $N$-Boc-activated monoterpene-fused $\beta$-lactam 3 were readily convertible to $N$-Boc $\beta$-amino acid 4, $\beta$-amino ester 7, and carboxamide derivatives 9-11 via nucleophilic attack on the activated lactam bond. The corresponding $\beta$-amino ester 7 was transformed to a novel amino acid 8.
\end{abstract}

Keywords: Stereoselective synthesis, $\beta$-lactam, $\beta$-amino acid, nucleophilic ring opening, enantiomers

\section{Introduction}

The readily available chiral terpenes and their derivatives are chiral auxiliaries that are widely used in enantioselective transformations. ${ }^{1-4}(+)$-Pulegon is a monoterpene frequently applied in asymmetric synthesis. As an example, the Eliel synthon (a 1,3-amino alcohol prepared from (+)pulegon) has been successfully utilized for the enantioselective synthesis of primary and secondary amines, $\alpha$-hydroxy acids, isoindolines, etc. ${ }^{5-8} \alpha$-Pinene is also a useful chiral source because it undergoes various transformations and both of its enantiomers are commercially available. Its derivatives, such as 2-hydroxypinan-3-one ${ }^{9}$ and $\beta$-isopinocamphenyl-9borabicyclo[3.3.1]nonane (Alpine-Borane ${ }^{\circledR}$ ), ${ }^{10}$ have been widely employed as chiral reagents in asymmetric syntheses. The preparation and some synthetic applications of optically pure 3amino-2-hydroxypinane have been reported by different authors. ${ }^{11,12}$ Monoterpene-fused 1,3oxazines have also been used as catalysts for enantioselective allylic substitution. ${ }^{13}$ In an earlier work, we reported the transformations of enantiomerically pure $\alpha$-pinene to monoterpene-fused saturated 1,3-heterocycles. ${ }^{14}$

$\beta$-Amino acids and their derivatives, such as amino esters, amides or 1,3-amino alcohols, can serve for the synthesis of a wide range of saturated heterocycles. ${ }^{15-17}$ They can also be used as 
building blocks in modified analogues of pharmacologically active peptides. ${ }^{18-20}$ Conformational studies of $\beta$-amino acid oligomers are also currently at the focus of interest. ${ }^{21-24}$ Besides their diverse chemical compositions, $\beta$-amino acids and their derivatives possess noteworthy pharmacological effects; for example, $(1 R, 2 S)$-2-aminocyclopentanecarboxylic acid (cispentacin) and some other alicyclic $\beta$-amino acids have marked antifungal activity. ${ }^{25}$

The present work describes a mild and efficient ring opening of monoterpene-fused $\beta$-lactam enantiomers derived from (+)- and (-)- $\alpha$-pinene, to produce a novel chiral $\beta$-amino acid and its derivatives.

\section{Results and Discussion}

The synthetic route for novel chiral $\beta$-amino acid derivatives is presented in Scheme 1. Even though the Schemes depict only compounds prepared from (-)-(1S,5S)- $\alpha$-pinene, all these reactions were performed by starting from both (-)-(1S,5S)- and (+)-(1R,5R)- $\alpha$-pinene (see Experimental Section). We have recently described the synthesis of the $\beta$-lactam enantiomers 2 from $\alpha$-pinene enantiomers by regiospecific and stereospecific addition ${ }^{26,27}$ of chlorosulfonyl isocyanate (CSI) (Scheme 1). ${ }^{14}$

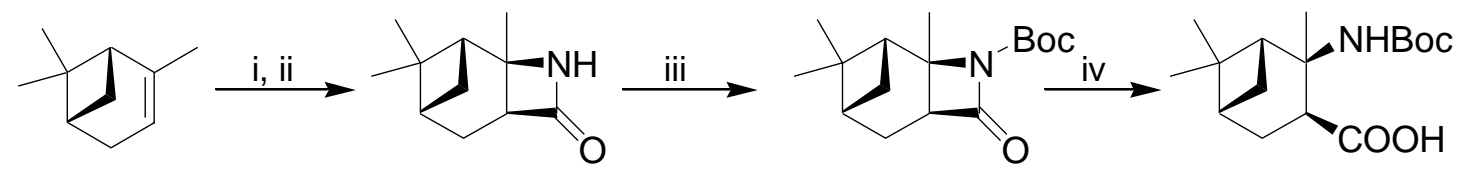

1

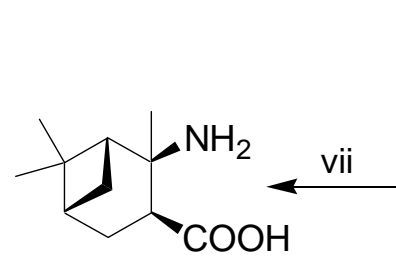

8
2

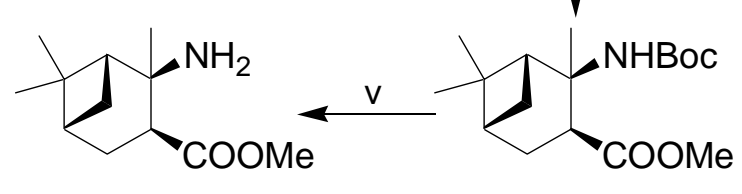

7
3

6

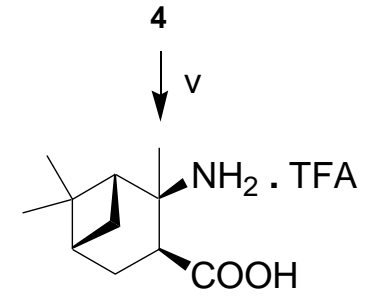

5

Scheme 1. (i) CSI, diethyl ether, $1 \mathrm{~h}$, rt., (ii) $\mathrm{Na}_{2} \mathrm{SO}_{3} / \mathrm{H}_{2} \mathrm{O}$, then $\mathrm{KOH}, 76 \%{ }^{14}$ (iii) (Boc) ${ }_{2} \mathrm{O}, \mathrm{Et}_{3} \mathrm{~N}$, DMAP/THF, rt., 12 h, 89\%; (iv) $\mathrm{LiOH} / \mathrm{H}_{2} \mathrm{O}$, rt., 7 h, 78\%; (v) TFA/ $\mathrm{CH}_{2} \mathrm{Cl}_{2}$, rt., 4 h, 96\%; (vi) cat. $\mathrm{NaOMe} / \mathrm{MeOH}$, rt., 0.5 h, 92\%; (vii) dioxane $/ \mathrm{H}_{2} \mathrm{O}$, reflux, 48 h, $85 \%$.

Although the literature includes several well-known methods for the ring opening of azetidinones, ${ }^{17}$ transformation of the azetidinone $\mathbf{2}$ to the amino acid by using aqueous hydrochloric acid solution failed. The synthesis of the amino ester by the refluxing of 2 with ethanolic $\mathrm{HCl}$ likewise resulted in the required ethyl ester only in low yield, with many sideproducts. ${ }^{14}$ The above results suggested that the strongly constrained pinane ring system is 
broken down under the highly acidic conditions necessary to open the $\beta$-lactam ring. Activation of the carboxamide bond of the azetidinone 2 therefore seemed necessary.

Treatment of the $\beta$-lactam 2 with di-tert-butyl dicarbonate resulted in the $N$-Boc $\beta$-lactam 3 , which was readily openable under mild conditions. The reaction of $\mathbf{3}$ with aqueous lithium hydroxide in THF gave the $N$-Boc $\beta$-amino acid 4 in good yield (78\%). ${ }^{28,29}$ Deprotection of 4 resulted in the TFA salt of the corresponding amino acid 8 in only $80 \%$ purity. Since the attempted purification of $\mathbf{5}$ failed, an alternative synthesis pathway was developed to prepare amino acid 8.

Ring opening of the $N$-Boc $\beta$-lactam 3 in the presence of a catalytic amount of $\mathrm{NaOMe}$ in dry $\mathrm{MeOH}$ gave 6 in excellent yield during a very short reaction time. ${ }^{30}$ Deprotection of 6 with TFA resulted quantitatively in 7 in high purity. The $\beta$-amino ester 7 was transformed to the $\beta$-amino acid 8 by refluxing in a dioxane:water $=1: 1$ mixture for 2 days (Scheme 1). ${ }^{31}$

The nucleophilic ring opening of the $N$-Boc $\beta$-lactam 3 was also performed with amines such as ammonia, methylamine, resulting in the $N$-Boc amides $\mathbf{9}$ and $\mathbf{1 0}$ in high yields. ${ }^{32}$ When benzylamine was applied transamidation process went through only in the presence of potassium cyanide as catalyst, resulting in amide 11 (Scheme 2$){ }^{29}$

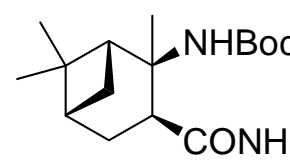

9,10

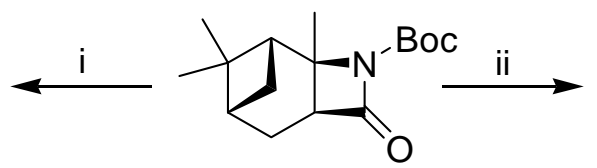

3

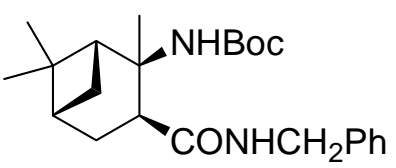

11

Scheme 2. (i) $\mathrm{R}=\mathrm{H}$ : $25 \% \mathrm{NH}_{3} / \mathrm{MeOH}, 4{ }^{\circ} \mathrm{C}, 12 \mathrm{~h}, 92 \%$; R $=\mathrm{Me}: 25 \% \mathrm{MeNH}_{2} / \mathrm{EtOH}, 4{ }^{\circ} \mathrm{C}, 12$ h, 86\%. (ii) $\mathrm{PhCH}_{2} \mathrm{NH}_{2}, \mathrm{KCN}, \mathrm{DMF}, 40{ }^{\circ} \mathrm{C}, 24$ h, $78 \%$.

The enantiomeric purity of the prepared compounds was proved by GC and NMR spectroscopy. There was no sign of the presence of any other diastereomer in the spectra of the prepared compounds.

\section{Conclusions}

An efficient method was devised for the ring opening of acid-sensitive monoterpene-fused $\beta$ lactams. The Boc-activated azetidinone 2 was found to be easily openable under mild conditions by nucleophilic attack. The novel $\beta$-amino acid $\mathbf{8}$ was also prepared. The resulting $\beta$-amino acid derivatives may serve as chiral building blocks in the asymmetric synthesis of potential pharmacons, $\beta$-amino acid oligomers and modified analogues of natural peptides. They are excellent starting materials for the preparation of potential chiral auxiliaries and catalysts such as 1,3-diamines and 1,3-amino alcohols which could be used in enantioselective syntheses. 


\section{Experimental Section}

General Procedures. ${ }^{1} \mathrm{H}$ NMR spectra were recorded on a Bruker Avance DRX 400 spectrometer (400 MHz, $\delta=0$ (TMS) in $\mathrm{CDCl}_{3}$, except for compound $\mathbf{8}$, which was dissolved in $\left.\mathrm{CD}_{3} \mathrm{OD}\right)$. Chemical shifts are expressed in ppm $(\delta)$ relative to TMS as internal reference. $J$ values are given in Hz. FT-IR spectra were recorded on a Perkin-Elmer model 1000 spectrophotometer. Microanalyses were determined on a Perkin-Elmer 2400 elemental analyser.

GC measurements were performed on a Chrompack CP-9002 system, consisting of a 901A Flame Ionization Detector and a Maestro II Chromatography data system (Chrompack International B.V., Middelburg, The Netherlands). The column used for direct separation was a CHIRASIL-DEX CB column (2500x0.25 mm I.D.) at $160{ }^{\circ} \mathrm{C}, 80 \mathrm{kPa}$ for 3 and 7. Optical rotations were obtained with a Perkin-Elmer 341 polarimeter. HRMS were recorded o a Finnigan MAT 95S instrument. Melting points were determined on a Kofler apparatus and are uncorrected. Azetidinone 2 was prepared from $(1 S, 5 S)-(-)-\alpha$-pinene by a literature method. ${ }^{14}$

$(1 R, 2 R, 5 S, 7 R)-N$-tert-Butoxycarbonyl-2,8,8-trimethyl-3-azatricyclo[5.1.1.0 $\left.{ }^{2,5}\right]$ nonan-4-one ${ }^{33}$ (3). To a stirred solution of azetidinone $2(5.0 \mathrm{~g}, 56 \mathrm{mmol})$ and dry THF $(100 \mathrm{~mL})$, triethylamine (1.60 mL, $112.0 \mathrm{mmol})$, di-tert-butyl dicarbonate $(18.34 \mathrm{~g}, 84.0 \mathrm{mmol})$ and a catalytic amount of 4-dimethylaminopyridine were added. After stirring for $12 \mathrm{~h}$ at room temperature (the reaction was monitored by means of TLC), the mixture was evaporated to dryness. The oily residue obtained was purified by flash chromatography on a silica gel column (hexane:ethyl acetate = 9:1), resulting in a white crystalline product $3(13.89 \mathrm{~g}, 89 \%$ yield $): \mathrm{mp} 94-96{ }^{\circ} \mathrm{C} ;[\alpha]_{\mathrm{D}}^{20}=+5.2(c$ $=0.2, \mathrm{MeOH}): \mathrm{IR}=775,1161,1307,1705,1793,2933 \mathrm{~cm}^{-1} .{ }^{1} \mathrm{H} \mathrm{NMR}\left(\mathrm{CDCl}_{3}\right) \delta(\mathrm{ppm}): 0.91$ $(3 \mathrm{H}, \mathrm{s}), 1.31(1 \mathrm{H}, \mathrm{d}, J=10.4 \mathrm{~Hz}), 1.32(3 \mathrm{H}, \mathrm{s}), 1.53(9 \mathrm{H}, \mathrm{s}), 1.65(3 \mathrm{H}, \mathrm{s}), 1.95-1.99(2 \mathrm{H}, \mathrm{m})$, 2.17-2.27 (2H, m), 2.40-2.44 (1H, m), $2.90(1 \mathrm{H}, \mathrm{d}, J=10.4 \mathrm{~Hz}) .{ }^{13} \mathrm{C} \mathrm{NMR}\left(\mathrm{CDCl}_{3}\right) \delta(\mathrm{ppm})$ : 23.7, 26.0, 26.4, 27.9, 28.7, 39.8, 41.9, 48.6, 50.7, 64.5, 83.2, 148.4, 171.2. Anal. Calcd. for $\mathrm{C}_{16} \mathrm{H}_{25} \mathrm{NO}_{3}$ (279.37): C, 68.79; H, 9.02; N, 5.01. Found: C, 68.89; H, 8.97; N, 5.17. HRMS calcd for $\mathrm{C}_{16} \mathrm{H}_{25} \mathrm{NO}_{3} 279.18344$, found 279.18362 .

The $1 S, 2 S, 5 R, 7 S$ enantiomer of 3 was prepared as described above; $[\alpha]_{\mathrm{D}}^{20}=-5.1(\mathrm{c}=0.2$, $\mathrm{MeOH})$; the spectroscopic data and $\mathrm{mp}$ were similar to those for 3. Analysis found: $\mathrm{C}, 68.85 ; \mathrm{H}$, $9.17 ; \mathrm{N}, 5.07$.

$(1 R, 2 R, 3 S, 5 R)$-2-tert-Butoxycarbonylaminopinane-3-carboxylic acid (4). The $N$-Boc lactam 3 $(3.0 \mathrm{~g}, 10.7 \mathrm{mmol})$ was dissolved in THF $(75 \mathrm{~mL})$ and treated with aq. LiOH $(1.8 \mathrm{~g}$ in $30 \mathrm{~mL}$ water) at room temperature. The mixture was stirred at room temperature for $7 \mathrm{~h}$. The THF was removed in vacuo, water $(15 \mathrm{~mL})$ was added, and the solution was acidified to $\mathrm{pH} 3.5-4.0$ with acetic acid and extracted with ethyl acetate $(3 \times 50 \mathrm{~mL})$. The combined organic phase was dried $\left(\mathrm{Na}_{2} \mathrm{SO}_{4}\right)$, and evaporated to give a colourless viscous oil $4(2.49 \mathrm{~g}, 78 \%$ yield $):[\alpha]_{\mathrm{D}}^{20}=+7.3(c=$ 0.40, MeOH): IR=1165, 1367, 1506, 1718, $2977 \mathrm{~cm}^{-1} .{ }^{1} \mathrm{H} \mathrm{NMR}\left(\mathrm{CDCl}_{3}\right) \delta(\mathrm{ppm}): 1.07(3 \mathrm{H}, \mathrm{s})$, $1.28(1 \mathrm{H}, \mathrm{d}, J=9.1 \mathrm{~Hz}), 1.29(3 \mathrm{H}, \mathrm{s}), 1.41(9 \mathrm{H}, \mathrm{s}), 1.64(3 \mathrm{H}, \mathrm{s}), 2.00-2.27(4 \mathrm{H}, \mathrm{m}), 2.55-2.58$ $(1 \mathrm{H}, \mathrm{m}), 3.15(1 \mathrm{H}, \mathrm{t}, J=9.1 \mathrm{~Hz}), 5.69(1 \mathrm{H}, \mathrm{bs}) .{ }^{13} \mathrm{C} \mathrm{NMR}\left(\mathrm{CDCl}_{3}\right) \delta(\mathrm{ppm}): 24.1,27.7,29.1$, 
29.5, 30.0, 31.4, 39.1, 40.6, 46.4, 52.8, 58.8, 79.3, 159.2, 180.1. Anal. Calcd. for $\mathrm{C}_{16} \mathrm{H}_{27} \mathrm{NO}_{4}$ (297.39): C, 64.62; H, 9.15; N, 4.71. Found: C, 64.89; H, 8.97; N, 4.56. HRMS calcd for $\mathrm{C}_{16} \mathrm{H}_{27} \mathrm{NO}_{4}$ 297.19401, found 297.19408.

The $1 S, 2 S, 3 R, 5 S$ enantiomer of 4 was prepared as described above; $[\alpha]_{\mathrm{D}}^{20}=-7.5(\mathrm{c}=0.40$, $\mathrm{MeOH})$; the spectroscopic data were similar to those for 4. Analysis found: C, 64.78; H, 9.01; N, 4.97.

Methyl $(1 R, 2 R, 3 S, 5 R)$-2-tert-butoxycarbonylaminopinane-3-carboxylate (5). To a stirred solution of $N$-Boc lactam $3(11.64 \mathrm{~g}, 42 \mathrm{mmol})$ in dry methanol $(60 \mathrm{~mL})$, NaOMe was added in a catalytic amount $(0.15 \mathrm{~g})$ at room temperature. After stirring for $2 \mathrm{~h}$ (the reaction was monitored by TLC), the reaction mixture was diluted with water $(250 \mathrm{~mL})$ and extracted with $\mathrm{CHCl}_{3}(3 \mathrm{x}$ $100 \mathrm{~mL})$. The combined organic phase was dried $\left(\mathrm{Na}_{2} \mathrm{SO}_{4}\right)$ and evaporated. A yellow oil was formed which gave $5(11.87 \mathrm{~g}, 92 \%$ yield $\left.):[\alpha]_{\mathrm{D}}^{20}=+34(c=0.21, \mathrm{MeOH})\right)$. IR $=1170,1367$, 1503, 1724, 2936, $3413 \mathrm{~cm}^{-1} .{ }^{1} \mathrm{H}$ NMR $\left(\mathrm{CDCl}_{3}\right) \delta(\mathrm{ppm}) 1.06(3 \mathrm{H}, \mathrm{s}), 1.27(1 \mathrm{H}, \mathrm{d}, J=10.6 \mathrm{~Hz})$, $1.29(3 \mathrm{H}, \mathrm{s}), 1.39(9 \mathrm{H}, \mathrm{s}), 1.61(3 \mathrm{H}, \mathrm{s}), 1.95-2.26(4 \mathrm{H}, \mathrm{m}), 2.56(1 \mathrm{H}, \mathrm{t}, J=5.5 \mathrm{~Hz}), 3.12(1 \mathrm{H}, \mathrm{t}, J$ $=9.6 \mathrm{~Hz}), 3.73(3 \mathrm{H}, \mathrm{s}), 5.53(1 \mathrm{H}, \mathrm{bs}) .{ }^{13} \mathrm{C} \mathrm{NMR}\left(\mathrm{CDCl}_{3}\right) \delta(\mathrm{ppm}): 24.1,27.5,29.1,29.4,29.9$, 31.6, 39.1, 40.7, 46.4, 52.6, 52.7, 58.5, 79.2, 155.1, 176.6. Anal. Calcd. for $\mathrm{C}_{17} \mathrm{H}_{29} \mathrm{NO}_{4}(311.42)$ : C, 65.57; H, 9.39; N, 4.50. Found: C, 65.41; H, 9.53; N, 4.84. HRMS calcd for $\mathrm{C}_{17} \mathrm{H}_{29} \mathrm{NO}_{4} 311.20966$, found 311.20829.

The $1 S, 2 S, 3 R, 5 S$ enantiomer of 5 was prepared as described above; $[\alpha]_{\mathrm{D}}^{20}=-34(\mathrm{c}=0.2$, $\mathrm{MeOH}$ ); the spectroscopic data were similar to those for 5 . Analysis found: $\mathrm{C}, 65.76 ; \mathrm{H}, 9.11$; N, 4.65.

Methyl $(1 R, 2 R, 3 S, 5 R)$-2-aminopinane-3-carboxylate (7). To a stirred solution of $N$-Boc amino ester $5(1.0 \mathrm{~g}, 3.2 \mathrm{mmol})$ in dry $\mathrm{CH}_{2} \mathrm{Cl}_{2}(50 \mathrm{~mL})$, trifluoroacetic acid $(2.5 \mathrm{~mL})$ was added at $0{ }^{\circ} \mathrm{C}$. After stirring for $4 \mathrm{~h}$, the solution was neutralized with ice-cold saturated aq. $\mathrm{NaHCO}_{3}$ solution and extracted with $\mathrm{CH}_{2} \mathrm{Cl}_{2}(3 \times 150 \mathrm{~mL})$. The combined organic phase was dried $\left(\mathrm{Na}_{2} \mathrm{SO}_{4}\right)$ and evaporated to give 6 as a colourless oil (130 mg, 96\% yield), which was used in the next step without futher purification: $[\alpha]_{\mathrm{D}}^{20}=+15.9(c=0.31, \mathrm{MeOH}), \mathrm{IR}=1165,1369,1735,2947 .{ }^{1} \mathrm{H}$ NMR $\left(\mathrm{CDCl}_{3}\right) \delta(\mathrm{ppm}): 1.02(3 \mathrm{H}, \mathrm{s}), 1.24(1 \mathrm{H}, \mathrm{d}, J=10.7 \mathrm{~Hz}), 1.26(3 \mathrm{H}, \mathrm{s}), 1.37(3 \mathrm{H}, \mathrm{s}), 1.68$ (1H, br s), 1.79-1.98 (3H, m), 2.20-2.36 (2H, m), $3.03(1 \mathrm{H}, \mathrm{dd}, J=6.9,10.1), 3.73(3 \mathrm{H}, \mathrm{s}) .{ }^{13} \mathrm{C}$ NMR $\left(\mathrm{CDCl}_{3}\right) \delta(\mathrm{ppm}): 24.0,28.3,28.6,29.3,31.4,39.7,40.3,46.7,51.8,55.6,56.2,175.9$. Anal. Calcd. for $\mathrm{C}_{12} \mathrm{H}_{21} \mathrm{NO}_{2}$ (211.30): C, 73.04; H, 11.75; N, 7.10. Found: C, 72.81; H, 11.93; N, 6.95. HRMS calcd for $\mathrm{C}_{12} \mathrm{H}_{21} \mathrm{NO}_{2} 211.15723$, found 211.15698 .

The $1 S, 2 S, 3 R, 5 S$ enantiomer of 7 was prepared as described above; $[\alpha]_{\mathrm{D}}^{20}=-16.2(\mathrm{c}=0.3$, $\mathrm{MeOH}$ ); the spectroscopic data were similar to those for 7. Analysis found: $\mathrm{C}, 73.24 ; \mathrm{H}, 11.55$; N, 7.02.

$(1 R, 2 R, 3 S, 5 R)$-2-Aminopinane-3-carboxylic acid (8). Amino ester 7 (1.1 g, $5.2 \mathrm{mmol})$ was dissolved in a mixture of dioxane and water $(1: 1,30 \mathrm{~mL})$. After stirring and reflux for 2 days (the reaction was monitored by means of TLC), the mixture was evaporated to dryness and the resulting white crystalline product 8 was filtered off and washed with acetone $(0.87 \mathrm{~g}, 85 \%$ yield): $\operatorname{mp~} 243-247{ }^{\circ} \mathrm{C} ;[\alpha]_{\mathrm{D}}^{20}=+16(c=0.2, \mathrm{MeOH}) . \mathrm{IR}=1381,1566,2931,3428 .{ }^{1} \mathrm{H}$ NMR 
$\left(\mathrm{CD}_{3} \mathrm{OD}\right) \delta(\mathrm{ppm}): 1.13(3 \mathrm{H}, \mathrm{s}), 1.27(1 \mathrm{H}, \mathrm{d}, J=10.9 \mathrm{~Hz}), 1.35(3 \mathrm{H}, \mathrm{s}), 1.53(3 \mathrm{H}, \mathrm{s}), 2.05-2.14$ $(3 \mathrm{H}, \mathrm{m}), 2.32-2.42(2 \mathrm{H}, \mathrm{m}), 2.96(1 \mathrm{H}, \mathrm{dd}, \mathrm{J}=8.3,10.2 \mathrm{~Hz}) .{ }^{13} \mathrm{C} \mathrm{NMR}\left(\mathrm{CD}_{3} \mathrm{OD}\right) \delta(\mathrm{ppm}): 23.7$, 28.4, 28.7, 29.1, 32.7, 40.3, 41.4, 45.2, 53.6, 59.9, 178.7. Anal. Calcd. for $\mathrm{C}_{11} \mathrm{H}_{19} \mathrm{NO}_{2}(197.27)$ : C, 66.97; H, 9.71; N, 7.10. Found: C, 67.17; H, 9.55; N, 7.23. HRMS calcd for $\mathrm{C}_{11} \mathrm{H}_{19} \mathrm{NO}_{2}$ 197.14158, found 197.14136.

The $1 S, 2 S, 3 R, 5 S$ enantiomer of 8 was prepared as described above; $[\alpha]_{\mathrm{D}}^{20}=-17(\mathrm{c}=0.2$, $\mathrm{MeOH})$; the spectroscopic data and mp were similar to those for 8. Analysis found: $\mathrm{C}, 66.81$; 9.93; N, 7.26.

tert-Butyl $\quad(1 R, 2 R, 3 S, 5 R)$-[(3-aminocarbonyl)-2,6,6-trimethylbicyclo[3.1.1]hept-2-yl]-carbamate (9). The $N$-Boc $\beta$-lactam $3(2.0 \mathrm{~g}, 7.17 \mathrm{mmol})$ was dissolved in a $25 \%$ solution of ammonia in dry methanol $(50 \mathrm{~mL})$. The reaction mixture was allowed to stand at $4{ }^{\circ} \mathrm{C}$ for $12 \mathrm{~h}$. After evaporation (first at room temperature and then on a $60{ }^{\circ} \mathrm{C}$ water bath), the resulting colourless oily product was purified by flash chromatography on a silica gel column (hexane:ethyl acetate $=4: 1)$ to give $9\left(1.95 \mathrm{~g} ; 92 \%\right.$ yield): $[\alpha]_{\mathrm{D}}^{20}=+33(c 0.2, \mathrm{MeOH}), \mathrm{IR}=$ 1065, 1170, 1511, 1672, 1718, 2979, $3379 \mathrm{~cm}^{-1} .{ }^{1} \mathrm{H}$ NMR $\left(\mathrm{CDCl}_{3}\right) \delta(\mathrm{ppm}): 1.06(3 \mathrm{H}, \mathrm{s}), 1.42$ $(3 \mathrm{H}, \mathrm{s}), 1.42(1 \mathrm{H}$, overlapping d), $1.48(9 \mathrm{H}, \mathrm{s}), 1.99-2.23(4 \mathrm{H}, \mathrm{m}), 2.54-2.57(1 \mathrm{H}, \mathrm{m}), 2.68-2.72$ $(1 \mathrm{H}, \mathrm{m}), 5.83(2 \mathrm{H}$, br d, $J=45.5 \mathrm{~Hz}), 6.33\left(1 \mathrm{H}\right.$, br s). ${ }^{13} \mathrm{C} \mathrm{NMR}\left(\mathrm{CDCl}_{3}\right) \delta(\mathrm{ppm}): 24.2,25.9$, 27.6, 28.3, 29.5, 39.9, 40.8, 47.6, 51.7, 52.8, 58.0, 79.1, 155.3, 177.7. Anal. Calcd. for $\mathrm{C}_{16} \mathrm{H}_{28} \mathrm{~N}_{2} \mathrm{O}_{3}$ (296.41): C, 71.95; H, 8.05; N, 9.32. Found: C, 71.79; H, 7.91; N, 9.45. HRMS calcd for $\mathrm{C}_{16} \mathrm{H}_{28} \mathrm{~N}_{2} \mathrm{O}_{3} 296.20999$, found 296.20947.

The $1 S, 2 S, 3 R, 5 S$ enantiomer of 9 was prepared as described above; $[\alpha]_{\mathrm{D}}^{20}=-33(\mathrm{c}=0.2$, $\mathrm{MeOH}$ ); the spectroscopic data were similar to those for 9. Analysis found: C, $72.12 ; \mathrm{H}, 8.23 ; \mathrm{N}$, 9.15 .

tert-Butyl (1R,2R,3S,5R)-[(3-methylaminocarbonyl)-2,6,6-trimethylbicyclo[3.1.1]hept-2-yl]carbamate (10). The $N$-Boc $\beta$-lactam $3(3.0 \mathrm{~g}, 10.78 \mathrm{mmol})$ was dissolved in a $25 \%$ solution of methylamine in dry methanol $(50 \mathrm{~mL})$. The reaction mixture was allowed to stand at $4{ }^{\circ} \mathrm{C}$ for 12 h. After evaporation, the crude yellow product was purified by flash chromatography on a silica gel column (hexane:ethyl acetate $=4: 1)$ to give 10 as white crystals $(1.82 \mathrm{~g} ; 86 \%$ yield): mp 81$85^{\circ} \mathrm{C} ;[\alpha]_{\mathrm{D}}^{20}=+14(c=0.23, \mathrm{MeOH}) . \mathrm{IR}=1365,1509,1662,1693,3349 .{ }^{1} \mathrm{H} \mathrm{NMR}\left(\mathrm{CDCl}_{3}\right) \delta$ (ppm): 1.04 (3H, s), $1.01(3 \mathrm{H}, \mathrm{s}, \mathrm{Me}-7), 1.28(3 \mathrm{H}, \mathrm{s}), 1.31(1 \mathrm{H}, \mathrm{d}, J=10.1 \mathrm{HZ}), 1.38(9 \mathrm{H}, \mathrm{s})$, $1.57(3 \mathrm{H}, \mathrm{s}), 1.96-2.03(2 \mathrm{H}, \mathrm{m}), 2.13-2.24(2 \mathrm{H}, \mathrm{m}), 2.67-2.75(2 \mathrm{H}, \mathrm{m}), 2.85(3 \mathrm{H}, \mathrm{d}, J=5.0 \mathrm{~Hz})$, $5.96\left(1 \mathrm{H}\right.$, br s), $6.51\left(1 \mathrm{H}\right.$, br s). ${ }^{13} \mathrm{C} \mathrm{NMR}\left(\mathrm{CDCl}_{3}\right) \delta(\mathrm{ppm}): 24.2,27.5,29.2,29.6,30.0,32.1$, 39.1, 40.9, 51.6, 58.1, 79.0, 155.3, 175.7. Anal. Calcd. for $\mathrm{C}_{17} \mathrm{H}_{30} \mathrm{~N}_{2} \mathrm{O}_{3}$ (310.43): C, 65.77; $\mathrm{H}$, 9.74; N, 9.02. Found: C, 65.95; H, 9.53; N, 9.17. HRMS calcd for $\mathrm{C}_{17} \mathrm{H}_{30} \mathrm{~N}_{2} \mathrm{O}_{3} 310.22564$, found 310.22558 .

The $1 S, 2 S, 3 R, 5 S$ enantiomer of $\mathbf{1 0}$ was prepared as described above; $[\alpha]_{\mathrm{D}}^{20}=-13.8(\mathrm{c}=0.21$, $\mathrm{MeOH})$; the spectroscopic data and mp were similar to those for 10. Analysis found: $\mathrm{C}, 65.98$; H, 9.63; N, 9.19. 
tert-Butyl (1R,2R,3S,5R)-[(3-benzylaminocarbonyl)-2,6,6-trimethylbicyclo[3.1.1]hept-2-yl]carbamate (11). The $N$-Boc $\beta$-lactam $3(0.84 \mathrm{~g}, 3.0 \mathrm{mmol})$ was dissolved in dry DMF (30 mL) under a $\mathrm{N}_{2}$ atmosphere, and $0.63 \mathrm{~g}(6.0 \mathrm{mmol})$ benzylamine and $0.15 \mathrm{~g}(2.3 \mathrm{mmol}) \mathrm{KCN}$ were added to the solution. After stirring for $24 \mathrm{~h}$ at $40{ }^{\circ} \mathrm{C}$ under a $\mathrm{N}_{2}$ atmosphere (the reaction was monitored by means of TLC), diethyl ether $(40 \mathrm{~mL})$ was added and the mixture was washed in turn with brine $(2 \times 40 \mathrm{~mL}), \mathrm{HCl}(1 \mathrm{~N}, 50 \mathrm{~mL})$ and saturated aqueous $\mathrm{NaHCO}_{3}(50 \mathrm{~mL})$. After drying $\left(\mathrm{Na}_{2} \mathrm{SO}_{4}\right)$ and evaporation of the organic phase, the white crystalline product obtained was purified by flash chromatography on a silica gel column (hexane: ethyl acetate $=4: 1$ ) to give 11 (0.29 g, 78\% yield): $\mathrm{mp}: 160-163{ }^{\circ} \mathrm{C} ;[\alpha]_{\mathrm{D}}^{20}=-19(c=0.21, \mathrm{MeOH}) . \mathrm{IR}=1253,1501,1637$, 1719, 2929, $3353 \mathrm{~cm}^{-1} .{ }^{1} \mathrm{H}$ NMR $\left(\mathrm{CDCl}_{3}\right) \delta(\mathrm{ppm}): 1.03(3 \mathrm{H}, \mathrm{s}), 1.29(3 \mathrm{H}, \mathrm{s}), 1.33(1 \mathrm{H}, \mathrm{d}, J=$ $10.6 \mathrm{~Hz}), 1.40(9 \mathrm{H}, \mathrm{s}), 1.59(3 \mathrm{H}, \mathrm{s}), 1.98-2.05(2 \mathrm{H}, \mathrm{m}), 2.20-2.26(2 \mathrm{H}, \mathrm{m}), 2.68-2.77(2 \mathrm{H}, \mathrm{m})$, $4.49(2 \mathrm{H}, \mathrm{ddd}, J=5.5,14.6,44.3 \mathrm{~Hz}), 6.01(1 \mathrm{H}$, br s$), 6.44(1 \mathrm{H}$, br s$), 7.29-7.36(5 \mathrm{H}, \mathrm{m}) .{ }^{13} \mathrm{C}$ $\operatorname{NMR}\left(\mathrm{CDCl}_{3}\right) \delta(\mathrm{ppm}): 24.2,27.7,29.2,29.5,30.0,32.0,39.1,40.9,44.7,48.8,51.7,58.2,79.0$, 128.3, 128.5, 129.4, 138.6, 155.3, 174.9. Anal. Calcd. for $\mathrm{C}_{23} \mathrm{H}_{34} \mathrm{~N}_{2} \mathrm{O}_{3}$ (386.53): C, 71.47; $\mathrm{H}$, 8.87; N, 7.25. Found: C, 71.59; H, 8.65; N, 7.43. HRMS calcd for $\mathrm{C}_{23} \mathrm{H}_{34} \mathrm{~N}_{2} \mathrm{O}_{3} 386.25694$, found 386.25731 .

The $1 S, 2 S, 3 R, 5 S$ enantiomer of 11 was prepared as described above; $[\alpha]_{\mathrm{D}}^{20}=+19(\mathrm{c}=0.20$, $\mathrm{MeOH})$; the spectroscopic data and mp were similar to those for 11. Analysis found: $\mathrm{C}, 71.61$; $\mathrm{H}, 8.65 ; \mathrm{N}, 7.33$.

\section{Acknowledgements}

Financial support from the National Scientific Research Foundation, Hungary (OTKA, F 032828 and TS 04888) is gratefully acknowledged.

\section{References and Notes}

1. Ho, T.-L. Enantioselective Synthesis of Natural Products from Chiral Terpenes; John Wiley, New York, 1992.

2. Steiner, D.; Sethofer, S.G.; Goralski, C.T; Singaram, B. Tetrahedron: Asymmetry 2002, 13, 1477.

3. Martínez, A.G.; Vilar, E.T.; Fraile, A.G.; de la Moya Cerero, S.; Martínez-Ruiz, P. Tetrahedron: Asymmetry 2002, 13, 1457.

4. Joshi, S.N.; Malhotra, S.V. Tetrahedron: Asymmetry 2003, 14, 1763.

5. He, X. C.; Eliel, E. L. Tetrahedron 1987, 43, 4979.

6. Pedrosa, R.; Andrés, C.; Nieto, J. J. Org. Chem. 2002, 67, 782.

7. Pedrosa, R.; Andrés, C.; Iglesias, J.M. Synlett 2002, 259.

8. Pedrosa, R.; Andrés, C.; de las Heras, L.; Nieto, J. Org. Lett. 2002, 4, 2513. 
9. Solladie-Cavallo, A.; Koessler, J. L. J. Org. Chem. 1994, 59, 3240.

10. Midland, M. M.; McDowell, D. C.; Hatch, R. L.; Tramontano, A. J. Am. Chem. Soc. 1980, $102,867$.

11. Rykowski, Z.; Wrzesien, J. Pol. J. Chem. 1981, 55, 371.

12. Masui, M.; Shioiri, T. Tetrahedron 1995, 51, 8363.

13. Evans, P.A.; Brandt, T.A. Tetrahedron Lett. 1996, 37, 9143.

14. Szakonyi, Z.; Martinek T.; Hetényi A.; Fülöp F. Tetrahedron: Asymmetry 2000, 11, 4571.

15. Juaristi, E., Ed.; Enantioselective Synthesis of $\beta$-Amino Acids; New York, 1997.

16. Fülöp, F. In Studies in Natural Product Chemistry; Atta-ur-Rahman, Ed.; "The chemistry of 2-aminocyclopentanecarboxylic acid.” Elsevier Science, 2000, Vol. 22, pp 273-306.

17. Fülöp, F. Chem. Rev. 2001, 101, 2181 and references cited therein.

18. Tóth, G. K.; Bakos, K.; Penke, B.; Pávó, L.; Varga, C.; Török, G.; Péter, A.; Fülöp, F. Bioorg. Med. Chem. Lett. 1999, 9, 667.

19. Steer, D. L.; Lew, R. A.; Perlmutter, P.; Smith, A. I.; Aquilar, M. I. Curr. Med. Chem. 2002, 9, 811.

20. Porter, E. A.; Wang, X.; Lee, H.; Weisblum, B.; Gellman, S. H. Nature 2000, 404, 565.

21. Abele, S.; Seebach, D. Eur. J. Org. Chem. 2000, 1.

22. Appella, D. H.; Christianson, L. A.; Karle, I. L.; Powell, D. R.; Gellman, S. H. J. Am. Chem. Soc. 1996, 118, 13071.

23. Martinek, T. A.; Tóth, G. K.; Vass, E.; Hollósi, M.; Fülöp, F. Angew. Chem., Int. Ed. Engl. 2002, 41, 1718.

24. Raguse, T. L.; Lai, J. R.; Gellman, S. H. J. Am. Chem. Soc. 2003, 125, 5592.

25. Mittendorf, J., Kunisch, F.; Matzke, M.; Militzer, H-C.; Schmidt, A.; Schönfeld, W. Bioorg. Med. Chem. Lett. 2003, 13, 433.

26. Kamal, A.; Sattur, P. B. Heterocycles 1987, 26, 1051.

27. Szakonyi, Z.; Fülöp, F.; Bernáth, G.; Evanics, F.; Riddell, F. G. Tetrahedron 1998, 54, 1013.

28. Furet, P.; García-Ecchevería, C.; Gay, B.; Schoepfer, J.; Zeller, M.; Rahuel, J. J. Med. Chem. 1999, 42, 2358.

29. Cundy, D. J.; Donohue, A. C.; McCarthy, T. D. J. Chem. Soc., Perkin Trans. 1 1999, 559.

30. Ojima, I.; Wang, T.; Delaloge, F. Tetrahedron Lett. 1998, 39, 3663.

31. Tamagnan, G.; Gao, Y.; Baldwin, R. M.; Zoghbi, S. S.; Neumeyer, J. L. Tetrahedron Lett. 1996, 37, 4353 .

32. Csomós, P.; Bernáth, G.; Fülöp, F. Monatsh.Chem. 2002, 133, 1077.

33. Although the transformation does not give rise to any change in the $(1 S, 5 S)$ configuration of (-)- $\alpha$-pinene, the configuration of the corresponding atoms in the products 3-11 is $(R, R)$, in consequence of the changes in CIP priority. 